\title{
Randomization of Node Scheme with Optimization in Wireless Sensor Network
}

\author{
Varsha
}

Research Scholar, Computer Science engineering, IKG Punjab Technical University, Kapurthala 144601,(Punjab) India barkhabright@gmail.com

Manju Bala

Khalsa College of Engineering \& Technology, Amritsar 143001, (Punjab), India. drmanju571@gmail.com

Manoj Kumar

DAV institute of Engineering, Management \& Technology, Jalandhar 144001 (Punjab), India

drmanojkumarindia@gmail.com

ABSTRACT-

Swarm Intelligence has nature inspired intelligence dependent on aggregate conduct of swarms having self-sorted out nature. Different techniques are being planned as far as ACO, PSO, Fish Swarm, Bats Swarm, Bacterial Foraging, TABU search and so forth.TABU search is regarded as heuristic method derived by Glover in 1986 depends on the memory structure. TABU search is used to determine the engineering design problems having continuous and real number variables. TABU is utilized to take care of different discrete issues in various regions of sciences and engineering. Since its advancement TABU has pulled in loads of specialists to take up its calculations and apply to take care of different complex issues and has been demonstrated the best strategy to get enhanced outcomes. The objective of this research paper is to implement TABU search to make the protocol more efficient and effective. This paper proposed MSEEC (multilevel stable and energy efficient clustering protocol) utilizing TABU mechanism in which normal nodes are randomly changed after each round in the territory of $200 \mathrm{~m} \times 200 \mathrm{~m}$. The recreation is done under the MATLAB environment and observed the performance of TABUMSEEC against MSEEC protocol on $4 \%$ increase in the case of first node dead (FND) and $20 \%$ increase in the case of last node dead (LND) and average remaining energy is delayed by $12 \%$ in advance nodes and $20 \%$ in super nodes respectively.

Keywords-Wireless sensor network, heterogeneity, TABUmechanism, MATLAB, FND, LND and average remaining energy.

\section{Introduction}

$\mathrm{W}$ reless sensor network (WSN) comprises of the unavoidable/pervasive computing. With the progression of assembling and remote innovations, numerous attainable applications are proposed, for example, modern sensor systems, habitat monitoring and so forth. The heterogeneous WSN contains sensor nodes with different limits, for instance, distant sensor types and correspondence/distinguishing extend, appropriately gives more noteworthy flexibility in organization.For example, we can build up a WSN in which nodes are outfitted with different sorts of sensors to give distinctive distinguishing organizations. The WSN comprise of two sorts of sensor nodes the first is having more lifetimes and all the more identifying area; the low-end ones are significantly more affordable and with compelled count and correspondence/recognizing limits. An adjustment plan of these nodes can achieve a leveling of execution like a couple of lower-end sensor nodes can be used to displace first class ones without reducing the lifetime of WSN.Numerous look into works have been proposed to address the sending issue of heterogeneous WSN. To achieve a fabulous execution, the circulation of heterogeneous WSN is more entangled than homogeneous WSN [1]. Distribution of nodes is fundamental already authentic establishment of sensor nodes, since various setups can be tried without thinking about the expense of genuine node distribution. Nonetheless, to emulate the conduct of WSN accurately is a noteworthy test of sensor nodes sending reproduction. HWSNs are especially valuable in genuine arrangements since they are all the more near genuine circumstances. Routing is a great challenge testing issue in the structure of WSNs. The stable and energy efficient clustering protocol (SEEC) have two highlights like it is energy proficiency situated convention and stability oriented protocol. In SEEC convention the entire system is partitioned into several different clusters. Every cluster has strong advance nodes $(\mathrm{AN})$ and $(\mathrm{NN})$ normal nodes are

conveyed arbitrarily in the system[2,3]. The normal nodes go about as detecting specialists and advance nodes collect information and send it to the sink. Then again, multilevel stable and energy efficient clustering protocol is utilized which is an expansion over the SEEC convention. In this convention, normal nodes (NN), advance nodes(AN) and super nodes (SN) are utilized dependent upon their underlying energy. Advance nodes having $\alpha$ time more energy and super nodes acquiring $\beta$ times more energy in comprise of normal nodes. The individuals from cluster will send information to advance and super head. After getting the information, the aggregation operation will be 
started which is reducing the duplicity of the information in the network. This convention is an upgrade over SEEC convention since it has progressively unstable duration. Since it is energy proficient convention however it experiences the heterogeneity of a protocol. If the Super Node(SN) which go about as a Cluster $\operatorname{Head}(\mathrm{CH})$ is die, the entire Network will goes out and Normal Node will coordinate Communicate to Advanced Node(AN) and energy Consumption is so high which will lessen the Network Lifetime of a Network. The Whole Network is Static and it additionally Causes Flooding at sink.

The Contribution to this paper is to enhance the MSEEC using TABU search mechanism has been developed for solving optimization problems for higher level heuristic procedure. It has the ability to find out the optimal and near optimal solution to problems. This paper addresses the matter of routing in WSNs using TABU search with the aim of maximize the network lifetime. Under contribution this paper proposes the TABUmultilevel stable and energy efficient clustering protocol (TABU-MSEEC) for the purpose of routing in WSN with the aim of maximize the network lifetime in the area of $200 \mathrm{~m} \times 200 \mathrm{~m}$.

Remaining paper is in following order: Section 2 elaborates the contribution and Section 3 formulates the related work. Section 4 defines the TABU-MSEEC. Section 5 depicts the TABU search algorithm and section 6 specifies the experimental setup and Section 7 defines the simulation Results and discussion and at last Section 8 formulate with conclusion and future scope.

\section{II.Contributions}

Following objectives have been considered for the advancement of multilevel stable and energy efficient clustering protocol:

- To enhance multilevel stable and energy efficient clustering protocol (MSEEC) over energy utilization by formulating significant criteria for routing.

- For uniform energy utilization over the system, another strategy for information transmission over the system is proposed.

\section{Related Works}

Many of the researches agilities in the situation of establishing a routing algorithm for WSNs are given in this section. The authors in [2015][4]generated the comparison among TSRA with different types of ACO. The results showed more balanced transmission between the nodes, reduces the cost as well as the energy consumption with extended the lifetime of network. The author has failed to upgrade the area for lifetime of network. In [2015][5]the author produced the metaheuristic algorithm based on TABU-search to give the optimal solution. The approach is based on greedy and random selection techniques. The work is carried out with QOI (Quality of information) with energy aware mobile sensing (QEMSS) problem but failed to explain the energy consumption of the nodes with minimization of sensing time. The author [2016][6]depicted the meta-heuristic techniques which are applied on combinatorial problem.
This technique relieves local search method from local optima problem and found the solution from single point which is fitted in all the aspects of computer science including VANET, software development effort estimation etc but has not implemented with TABU. The author[2017][7] developed the TABU search approach for better combination of weights to be allocated with the trust factors in order to find the trusted sensor operations. The results approved the reduction of search space by $22 \%$ in comparison to the local search having simulated annealing (SA). The author [2017][8]found difficulty for global optimum in traditional optimization techniques. A novel Entanglement QTS is proposed which is based on the QTS (quantum-inspired TABU search) algorithm. The search discovered the optimal solution found the optimal local solution by quantum not gate and also managed the strongdependence problems and this approach proved to be better in terms of effectiveness at global optimum with its computational efficiency. The author[2018][9] depicted the meta-heuristic techniques based on PSO with TABU search algorithm as PSO is not effective to find local optima problem. The TABU search made the problem easier and enhanced the number of clusters with reduction in packet loss and end to end delay.

\section{TABU-multilevel stable and energy efficient clustering protocol}

This paper described TABU-MSEEC protocol for WSNs in the presence of node heterogeneity with the routing done by TABU search in the randomization of normal nodes against the area of $200 \mathrm{~m} \times 200 \mathrm{~m}$ using TABU search for MSEEC energy efficient clustering protocol[10]. In TABU-MSEEC, there are three types of nodes; normal node, advance node and super node based on their initial energy value. The position of advance nodes and super nodes are fixed but the position of normal nodes is changed with every round. $\mathrm{M}_{\mathrm{sn}}$ is the proportion of entire number of nodes having $\alpha$ times greater energy in comprise of normal nodes called super nodes. The remaining nodes having energy denotes by $\mathrm{E}_{0}$.

$\mathrm{P}=\mathrm{p} 1+\mathrm{p} 2$ where $\mathrm{p} 1$ is the entire number of normal nodes attached to advance nodes and $\mathrm{p} 2$ is the entire normal nodes attached to super nodes. The entire energy equation of three level nodes is given below:-

$E_{\text {total }}=p \cdot E_{0}+M_{\text {AN }} \cdot(1+\alpha) \cdot E_{0}+M_{\mathrm{sn}} \cdot(1+\beta) \cdot E_{0}$

Therefore, the three-level M-SEEC has $\left(\alpha \cdot \mathrm{M}_{\mathrm{AN}}+\beta \cdot \mathrm{M}_{\mathrm{SN}}\right)$ times more energy. From the figure 1, we can easily see the randomization of normal nodes. 


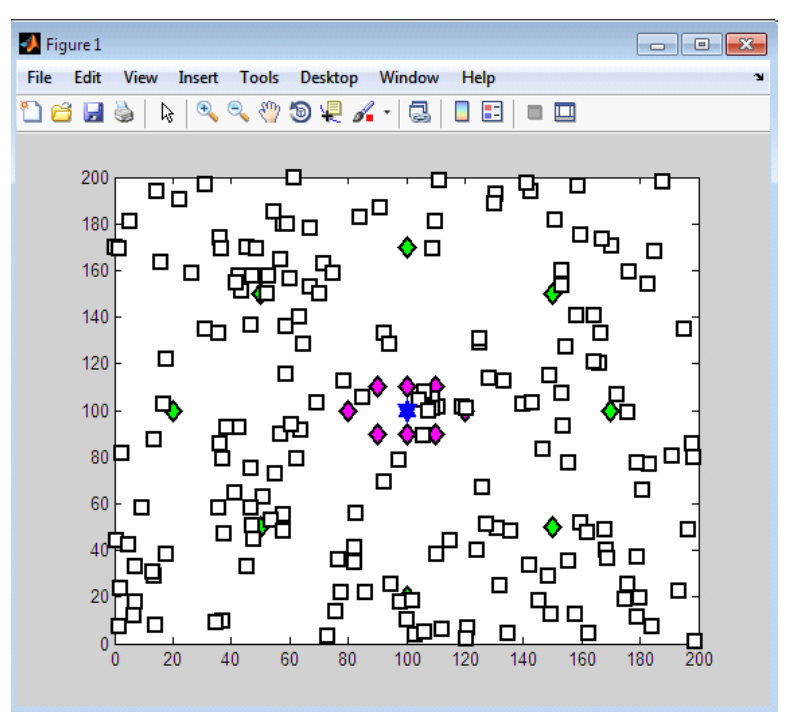

Figure 1.Snapshot of the entire network when 200 nodes are distributed in $200 \mathrm{~m} \times 200 \mathrm{~m}$ network.

\section{Level of heterogeneity}

The level of heterogeneity is represented by the given formula that is

$\mathrm{R}_{\mathrm{i}-1}=\mathrm{R}_{\mathrm{i}-2}$

Where i denotes the heterogeneity level.

\section{Optimal Number of ANs}

The measure of energy taken during a solitary round is expressed by following condition

$\mathrm{E}_{\mathrm{AN}}=$ nA. L. $\mathrm{E}_{\text {elec }}+$ nA. L. $\mathrm{E}_{\mathrm{DA}}+\mathrm{L} \cdot \mathrm{E}_{\mathrm{elec}}+$ L. $\mathrm{e}_{\mathrm{fs}}$.

Where EDArepresents the data aggregation cost and R1 represents the distance among advance node and base station. The Energy utilized by normal nodes are expressed by following equation

$\mathrm{E}_{\mathrm{NN}}=\mathrm{L} \cdot \mathrm{E}_{\mathrm{elec}}+$ L. $\mathrm{e}_{\mathrm{fs}}$.

Where $\mathrm{d}_{\mathrm{tO} \text { AN }}^{2}$ represent the distance between the normal node and advance nodes.

The total energy consumed during a single round is expressed by following condition

$\mathrm{E}_{\text {Round }}=\mathrm{m}_{\mathrm{AN}}\left(\frac{\mathrm{n} 1}{\mathrm{~m}_{\mathrm{AN}}} \cdot \mathrm{L} \cdot \mathrm{E}_{\mathrm{elec}}+\frac{\mathrm{n} 1}{\mathrm{~m}_{\mathrm{AN}}} \cdot \mathrm{L} \cdot \mathrm{E}_{\mathrm{DA}}+\mathrm{L} \cdot \mathrm{E}_{\mathrm{elec}}+\right.$ L. $\left.E_{\mathrm{fs}} \cdot R 1^{2}+\frac{\mathrm{n} 1}{\mathrm{~m}_{\mathrm{AN}}} \cdot \mathrm{L} \cdot \mathrm{E}_{\mathrm{elec}}+\frac{\mathrm{n} 1}{\mathrm{~m}_{\mathrm{AN}}} \cdot \mathrm{L} \cdot \mathrm{E}_{\mathrm{fs}} \cdot \mathrm{d}_{\mathrm{to}}^{2} \mathrm{AN}\right)$

After differentiating $\mathrm{E}_{\mathrm{Round}}$ correspond to $\mathrm{m}_{\mathrm{AN}}$ and equalizing with zero, the minimum number of advance nodes generated to be

$\mathrm{m}_{\mathrm{AN}}$ opt $=0.7668$

\section{Required energy for AN}

For computing the energy of advance nodes, firstly calculate the total lifetime of a network which is the total of all rounds from beginning to end.

Rounds $_{\mathrm{AN}}=\mathrm{AN}$ and

Rounds $_{\mathrm{NN}}=\mathrm{NN}$

The value of advance node should be greater than that of normal nodes in each cluster

$\alpha \geq\left[\frac{n_{A} \cdot \text { Eelec }+n_{A} E_{D A}+E_{e l e c}+E_{f s} \cdot R 1^{2}}{E_{\text {elec }}+E_{f s} \cdot d_{\text {to AN }}^{2}}\right]-1$

\section{Optimal Number of SNs}

The measure of energy taken during a solitary round is expressed by following condition

$\mathrm{E}_{\mathrm{SN}}=\mathrm{nS}$. L. $\mathrm{E}_{\mathrm{elec}}+\mathrm{nS}$. L. $\mathrm{E}_{\mathrm{DA}}+\mathrm{L} . \mathrm{E}_{\mathrm{elec}}+$ L. $\mathrm{e}_{\mathrm{fs}}$

Where EDA represents the data aggregation cost and R1 represents the distance among advance node and base station. The Energy utilized by normal nodes are expressed by following equation

$\mathrm{E}_{\mathrm{NN}}=\mathrm{L} \cdot \mathrm{E}_{\mathrm{elec}}+$ L. $\mathrm{e}_{\mathrm{fs}}$

Where $d_{\text {tO CH }}^{2}$ represent the distance among the normal node and its super node and advance nodes.

The total energy consumed during a single round is expressed by following condition

$\mathrm{E}_{\mathrm{Round}}=\mathrm{m}_{\mathrm{AN}}\left(\mathrm{E}_{\mathrm{AN}}+\mathrm{n}_{\mathrm{A}} \cdot \mathrm{E}_{\mathrm{NN}}+\mathrm{m}_{\mathrm{SN}} \cdot\left(\mathrm{E}_{\mathrm{SN}}+\mathrm{n}_{\mathrm{S}} \cdot \mathrm{E}_{\mathrm{NN}}\right)\right.$

After differentiating $E_{\text {Round }}$ correspond to $m_{\mathrm{SN}}$ and associating with zero, the minimum number of advance nodes found to be

$\mathrm{M}_{\mathrm{SN}}$ opt $=0.7668 \sqrt{\mathrm{n} 2}$

\section{Required energy for SN}

For computing the energy of advance nodes, firstly calculate the total lifetime of a network which is the total of all rounds from beginning to end.

Rounds $\mathrm{SN}=\mathrm{SN}$

and

Rounds NN = NN

The value of advance node should be greater than that of normal nodes in each cluster

$\boldsymbol{\beta} \geq\left[\frac{n_{S N} \cdot \text { Eelec }+\mathrm{n}_{S N} E_{D A}+E_{\text {elec }}+E_{f s} \cdot R 2^{2}}{E_{\text {elec }}+E_{f s} \cdot d_{\text {to } S N}^{2}}\right]-\mathbf{1}$

\section{TABU Search Algorithm}

Essentially, the TABU search was generated by Fred Glover and this instrument is enlivened by human memory with the end goal to enhance the productivity of the investigation procedure, one needs to store the neighborhood data (like the present estimation of the goal work) yet in addition of some data identified with the investigation procedure. To locate the ideal arrangement, the TABU search produces the underlying arrangement attainably. The underlying arrangement turns out to be at the same time the best arrangement and current arrangement and by and large this arrangement is embedded into the memory list, called TABU list. This list 
is utilized to keep the cycling. On the off chance that TABU list is full; the most established component of TABU list is evacuated and new is included [11,12]. The TABU scan acquired the area answers for the present arrangement, and updates the present arrangement with TABU list. In each time, the arrangement of neighbors of current arrangement is make by the area tasks and the neighbor with the most noteworthy esteem is check as the new best arrangement in TABU list and new best arrangement is acknowledged for the choice of hopeful arrangement, generally the arrangement having the following most astounding quality will turns into the competitor arrangement. Presently, the expense of new best arrangement is contrasted and the best arrangement generally cycles is increments and new best arrangement is updated into the TABU list and mentioned as the present answer for the next arrangement. The means of TABU search are characterized underneath:-

It could be communicated as seeks after $(i *$ is the best arrangement found up until now and $\mathrm{k}$ the cycle counter):

Stage 1. Pick the first solution $I$ in S. Set $i^{*}=i$ and $k=0$.

Stage 2. Set $\mathrm{k}=\mathrm{k}+1$ and create a subset $\mathrm{V}^{*}$ of arrangement in $\mathrm{N}(\mathrm{i}, \mathrm{k})$

Stage 3. Pick a best $\mathrm{j}$ in $\mathrm{V}^{*}$ (regarding $\mathrm{f}$ or to some altered capacity $\mathrm{f} \sim$ ) and set $\mathrm{i}=\mathrm{j}$.

Stage 4. In the event that $\mathrm{f}(\mathrm{i})<\mathrm{f}\left(\mathrm{i}^{*}\right)$ set $\mathrm{i}^{*}=\mathrm{i}$.

Stage 5. In the event that a ceasing condition is met, stop. Else go to Step 2.

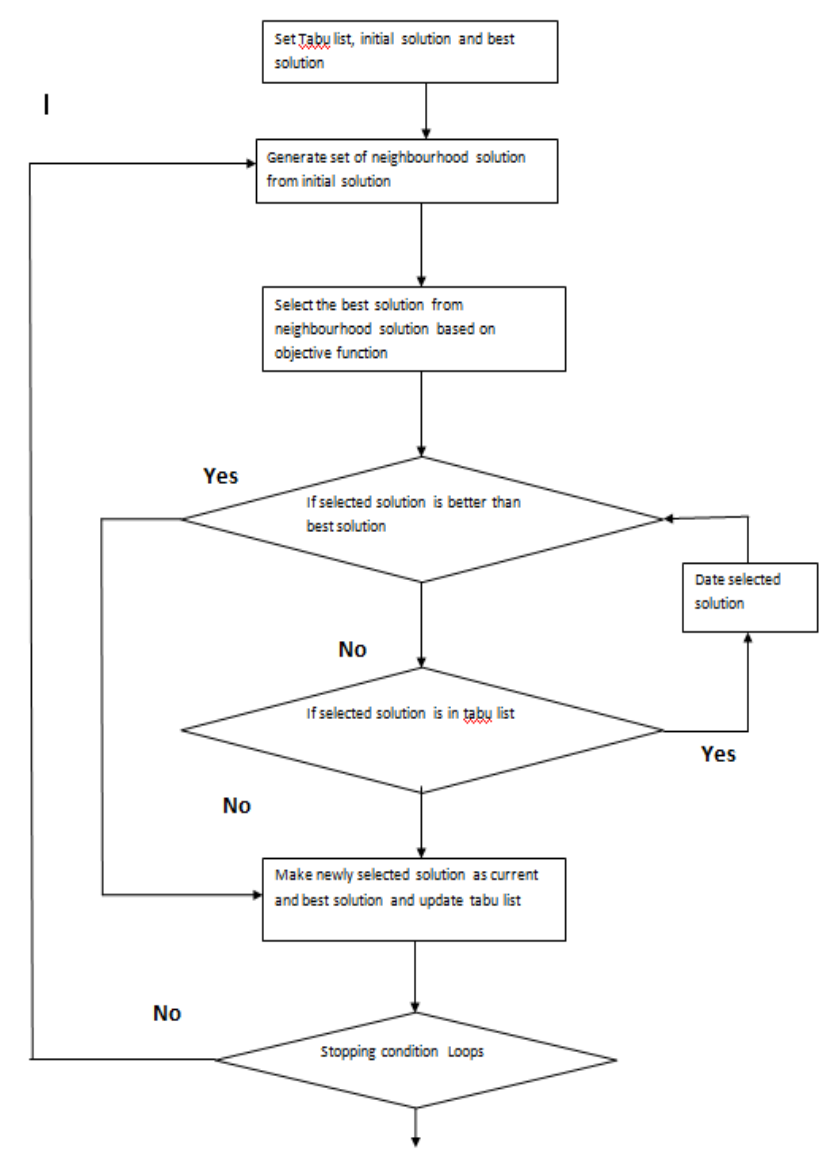

Figure 2. Flow chart ofTABU-SEARCH

Guidelines related to TABU SEARCH

- When TABU limitations depend on a solitary kind of move characteristic, it is commonly desirable over select a quality whose TABU status less unbendingly confines the decision of accessible moves.

- Multiple TABU records have been utilized essentially to keep up discrete records for various arrangement forms or to avert reiterations and additionally inversions.

- Embody the treatment of goal criteria in a credit based system practically equivalent to that used to characterize TABU limitations[13].

- To join the enhancement and increase objectives, make a rating framework by reference to target examination, keeping up records of profoundly evaluated characteristics and how regularly these qualities show up in arrangements produced.

- Devote a set number of primer cycles to distinguish verifiable measurements for move assessments in each separation class and apply connections from target investigation to get viable decisions at consequent emphases. [14] 
- For longer term expansion, utilize recurrence inferred punishments to get the pursuit far from arrangements recently experienced either by restarting or by dynamic from the present arrangement.

- As TABU search is higher level optimization technique so various applications of TABU search is mentioned with references.[15]

Table 1: Various Applications of TABU-Search

\begin{tabular}{|c|c|c|}
\hline Area & Brief description & Reference \\
\hline Scheduling & $\begin{array}{l}\text { Employed } \\
\text { scheduling } \\
\\
\text { Flow shop } \\
\text { scheduling }\end{array}$ & $\begin{array}{l}\text { Glover and } \\
\text { mcmillian [16] } \\
\text { Widmer and } \\
\text { hertz[17] }\end{array}$ \\
\hline Graph & Clustering & $\begin{array}{l}\text { Glover et al[18] } \\
\text { Hansen et al[19] } \\
\text { Dorndorf and } \\
\text { Pesch }\end{array}$ \\
\hline $\begin{array}{l}\text { Neural } \\
\text { network }\end{array}$ & $\begin{array}{l}\text { Learning in an } \\
\text { associative } \\
\text { memory } \\
\text { Nonconvex } \\
\text { optimization } \\
\text { problems }\end{array}$ & $\begin{array}{l}\text { De werra and } \\
\text { hertz[20] } \\
\text { Beyer and ogier } \\
{[21]}\end{array}$ \\
\hline
\end{tabular}

\section{Experimental Setup}

The MATLAB simulator is used for the implementation. In the first case, 200 nodes are placed in which 8 advanced nodes and 8 super nodes are fixed and rest of the normal nodes are changed after every round inside the area of $200 \times 200 \mathrm{~m}^{2}$. Here, the parameters are used in the simulation are mentioned below in table 2 .

Table 2:Network Parameters

\begin{tabular}{|l|l|}
\hline \multicolumn{1}{|c|}{ Parameter } & Value \\
\hline $\begin{array}{l}\text { Terrain of the } \\
\text { network }\end{array}$ & $200 \times 200$ \\
\hline Position of Sink & 100,100 \\
\hline $\begin{array}{l}\text { Total number of } \\
\text { Nodes }\end{array}$ & 200 \\
\hline $\begin{array}{l}\text { Probability(p) to be a } \\
\text { cluster head }\end{array}$ & 0.1 \\
\hline
\end{tabular}

\begin{tabular}{|l|l|}
\hline Startup Energy(Eo) & $0.5 \mathrm{~J}$ \\
\hline Transmiter_energy & $50 \mathrm{~nJ} / \mathrm{bit}$ \\
\hline Receiver_energy & $50 \mathrm{~nJ} / \mathrm{bit}$ \\
\hline Free space & $10 \mathrm{nj} / \mathrm{bit} / \mathrm{m}^{2}$ \\
\hline Multipath & $0.0013 \mathrm{pJ} / \mathrm{bit} / \mathrm{m}^{4}$ \\
\hline
\end{tabular}

\section{Simulation Results and Discussion}

On applying TABU search mechanism, the following results will come out. For simulation MATLAB 2013 a tool is used for easy computing of results. All results were compared with the basic MSEECand TABU- MSEEC.The performance metrics used in the results shown that the proposed TABU-MSEECgives the larger stability period, more efficient in favor of energy and high network lifetime than the MSEEC. In this case, we moving the normal nodes with each round and position of 8 advanced nodes and 8 super nodes are fixed against the area of $200 \mathrm{~m}$ $\times 200 \mathrm{~m}$. The stability period of TABU-MSEEC is much longer than that of other case. This paper observed that TABU MSEEC take some advantage in forms of death of first node and last node, which enhances the network stability.

\section{Number of dead nodes}

Figure 3depicts that in MSEEC network the first and last sensor node death is after 5100and 15000 rounds. However, for TABU MSEEC network the first node and last node dead is after 6000 and 20000 rounds respectively. From the results, it is clearly shown that the performance of TABU MSEEC is $4 \%$ raise in case of first node dead (FND) and 20\% raise in case of last node dead (LND). Certainly, this change in the performance is due to the modification in the simulation setup because the protocol has the random normal node mobility. Table 3 calculates the exact reading of the network.

Table 3: First node dead and Last node dead comparison among protocols

\begin{tabular}{|l|l|l|l|}
\hline Dead Nodes & Protocol & FND & LND \\
\hline $\begin{array}{l}\text { All nodes } \\
\text { including NN, }\end{array}$ & MSEEC & 5100 & 15000 \\
\cline { 2 - 4 } AN and SN & $\begin{array}{l}\text { TABU } \\
\text { MSEEC }\end{array}$ & 6000 & 20000 \\
\hline
\end{tabular}

Figure 3. Number of dead nodes vs. no of rounds for 200 nodes in $200 \times 200$ network.

\section{Number of alive nodes}

Figure 4 represents the number of alive nodes against the number of rounds. From the figure, it is clearly shown that the nodes are alive while increases the number of rounds. To evaluating the energy efficiency of a network, the 


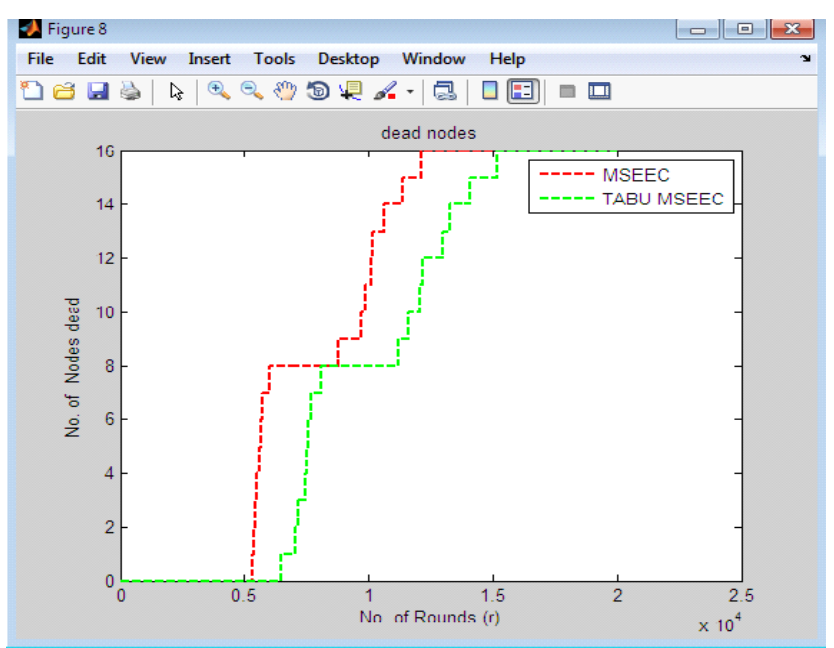

number of alive nodes was calculated for every round. From the simulation, it is observed that the alive nodes in case of MSEEC protocol are 12000 rounds and in case of TABU MSEEC it is 15000 . Proposed protocol has $12 \%$ improvement which shows that TABU MSEEC performs better than that of MSEEC protocol.Table 4 calculates the exact reading of the network.

Table 4: Readings of alive node with respect to rounds among different protocols

\begin{tabular}{|l|l|l|}
\hline Alive Nodes & Protocol & No of rounds \\
\hline $\begin{array}{l}\text { All nodes } \\
\text { including NN, }\end{array}$ & MSEEC & 12000 \\
\cline { 2 - 3 } AN and SN & TABU MSEEC & 15000 \\
\hline
\end{tabular}

Figure 4.Alive nodes vs. no of rounds for 200 nodes in $200 \times 200$ network.

Average remaining energy of normal node (NN), advance node (AN) and super node (SN):-

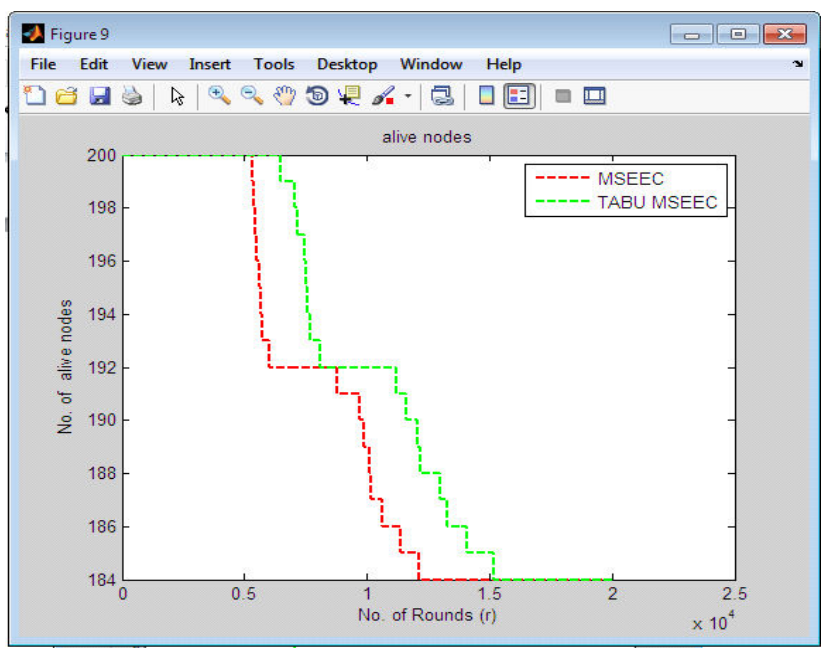

Figure $5(\mathrm{a}, \mathrm{b}, \mathrm{c})$ gives the average remaining energy in every transmission round. Average remaining energy of nodes should be calculated by this method.[22,23]
Suppose Ei be the initial energy of node and energy consumed by node $\mathrm{E}(\mathrm{t})$ after $\mathrm{t}$ time period is given by

$\mathrm{E}(\mathrm{t})=($ Number s_packet $* \alpha)+\left(\right.$ Number $\mathrm{r} \_$packet $\left.* \beta\right)$

Where, Number s_packet $=$ number of packet send,

Number r_packet $=$ number of packet receive

$(\alpha, \beta) €$ range $[0,1]$

Now, Erem of a node at time $t$ is calculated using the equation

$$
\mathrm{E}_{\mathrm{rem}}=\mathrm{E}_{\mathrm{i}}-\mathrm{E}(\mathrm{t})
$$

The average remaining energy is very important parameter in this model. So, the observations have to be made in case of normal node, advance node and super node with MSEEC and TABU-MSEEC protocol.

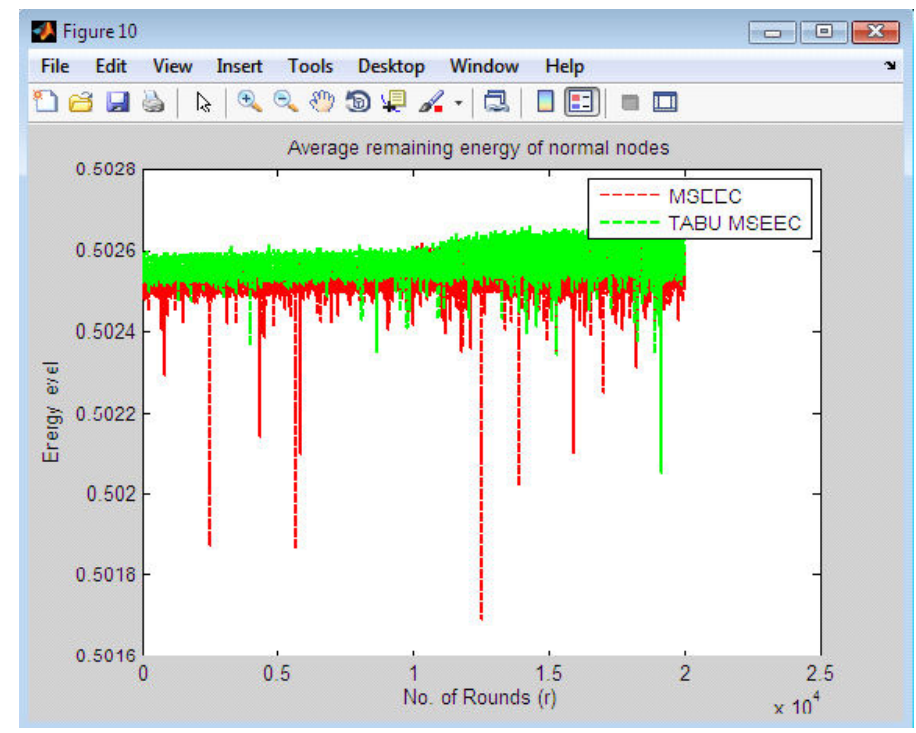

Figure 5. a)Average remaining energy of normal nodes vs. no of rounds for 200 nodes in $200 \times 200$ network.

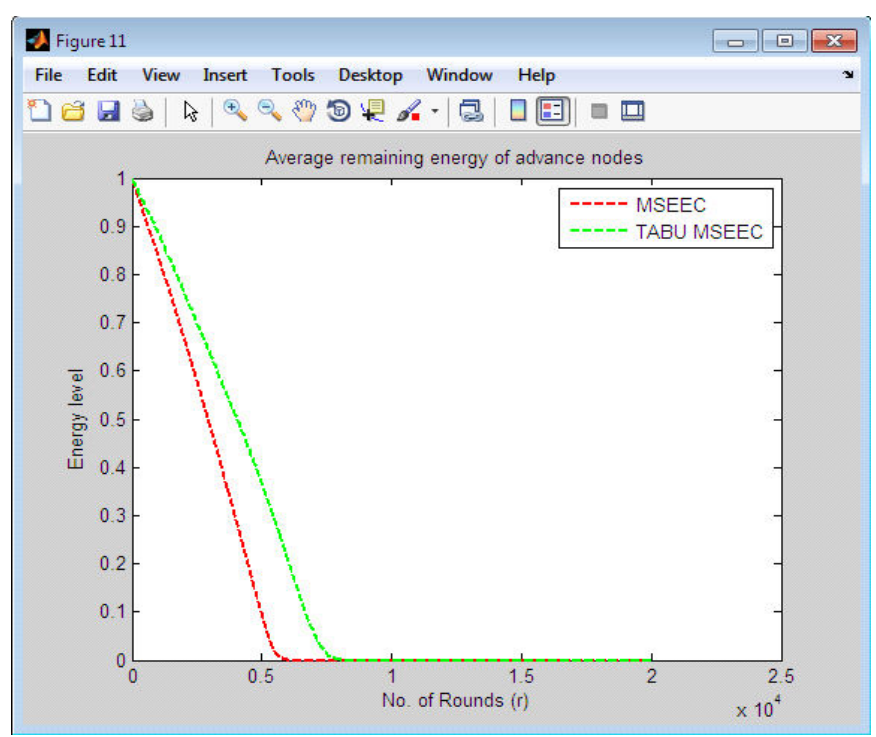

b) Average remaining energy of advance nodes vs. no of rounds for 200 nodes in $200 \times 200$ network. 


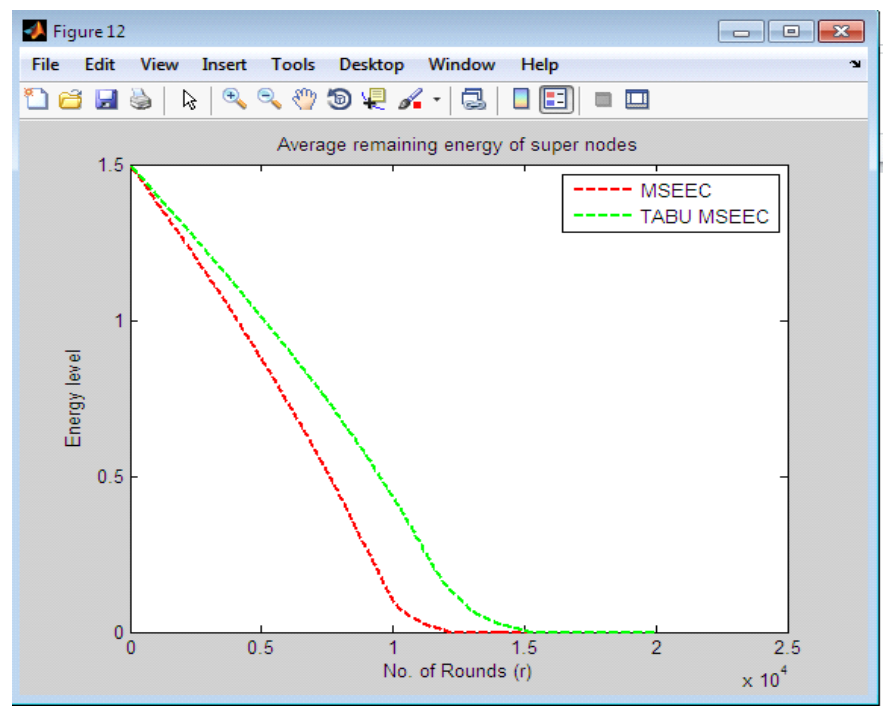

c)Average remaining energy of super nodes vs. no of rounds for 200 nodes in $200 \times 200$ network.

Here, we have observed the average remaining energy of nodes after particular rounds of simulation. The remaining energy of network degrades rapidly in MSEEC protocol. The average remaining energy represents a slope generally $0.5025 \mathrm{~J} /$ round in MSEEC, comparison to $0.5026 \mathrm{~J} /$ round in TABU MSEEC protocol respectively. In case of average remaining energy in advance nodes and in super nodes is upto 5100 and 10000 rounds while in case of TABU MSEEC it is 8000 and 15000 rounds respectively. So, it is clearly shown that the average remaining energy is delayed by $12 \%$ in advance nodes and $20 \%$ in super nodes respectively. Here, the observations reveal that the performance of TABU SEARCH is very high as compared to the MSEEC protocol because TABU search chooses the most effective solution.

\section{CONCLUSION AND FUTURE SCOPE}

In this paper, MSEEC and TABU MSEEC protocol with random mobility in nodes has been proposed for wireless sensor network. Here, the performance comparison is done and found that TABU-MSEEC is $4 \%$ raise in case of first node dead (FND) and $20 \%$ raise in case of last node dead (LND) and the average remaining energy is delayed by $12 \%$ in advance nodes and $20 \%$ in super nodes respectively. Here, the observations reveal that the performance of TABU SEARCH is very high as compared to the MSEEC protocol because TABU search chooses the most effective solution. As a future work, gathered data can be utilized to decide the impact of the path loss in applications that relies upon RSSI and path loss exponent, for example, RSS-based confinement and energy analysis and also work on clustering as well as routing with other bio-inspired algorithms like cuckoo search, flower pollination, spider monkey optimization, Glow-worm, Meer cat Clan algorithm.

\section{References}

[1] Farouk, F., Rizk, R., \& Zaki, F. W. (2014). Multi-level stable and energy-efficient clustering protocol in heterogeneous wireless sensor networks. IET Wireless Sensor Systems, 4(4), 159-169.

[2] Wang, X., Qian, L., Wu, J., \& Liu, T. (2010). An energy and distance based clustering protocol for wireless sensor networks. In Novel Algorithms and Techniques in Telecommunications and Networking (pp. 409-412). Springer, Dordrecht.

[3] Li, X., Li, N., Chen, L., Shen, Y., Wang, Z., \& Zhu, Z. (2010, March). An improved LEACH for clustering protocols in wireless sensor networks. In 2010 International Conference on Measuring Technology and Mechatronics Automation (Vol. 1, pp. 496-499). IEEE.

[4] Orojloo, H., \& Haghighat, A. T. (2016). A Tabu search based routing algorithm for wireless sensor networks. Wireless Networks, 22(5), 1711-1724.

[5] Messaoud, R. B., \& Ghamri-Doudane, Y. (2015, September). QoI and energy-aware mobile sensing scheme: A tabu-search approach. In 2015 IEEE 82nd Vehicular Technology Conference (VTC2015-Fall) (pp. 16). IEEE.

[6] Amuthan, A., \& Thilak, K. D. (2016, October). Survey on Tabu search meta-heuristic optimization. In 2016 International Conference on Signal Processing, Communication, Power and Embedded System (SCOPES) (pp. 1539-1543). IEEE.

[7] Habib, S. J., \& Marimuthu, P. N. (2017, April). Reputation analysis of sensors' trust within tabu search. In World Conference on Information Systems and Technologies (pp. 343-352). Springer, Cham.

[8] Kuo, S. Y., \& Chou, Y. H. (2017). Entanglementenhanced quantum-inspired tabu search algorithm for function optimization. IEEE Access, 5, 13236-13252.

[9] Vijayalakshmi, K., \& Anandan, P. (2018). A multi objective Tabu particle swarm optimization for effective cluster head selection in WSN. Cluster Computing, 1-8.

[10] Glover, F. (1990). Tabu search: A tutorial. Interfaces, 20(4), 74-94.

[11] de Werra, D., \& Hertz, A. (1989). Tabu search techniques. Operations-Research-Spektrum, 11(3), 131141.

[12] Vaithyanathan, S., Burke, L. I., \& Magent, M. A. (1996). Massively parallel analog tabu search using neural networks applied to simple plant location problems. European Journal of Operational Research, 93(2), 317330.

[13] Reinelt, G. (1994). The traveling salesman: computational solutions for TSP applications. SpringerVerlag.

[14] Gendreau, M., Laporte, G., \& Séguin, R. (1996). A tabu search heuristic for the vehicle routing problem with stochastic demands and customers. Operations Research, 44(3), 469-477.

[15] Kinney, G. W., Barnes, J. W., \& Colletti, B. W. (2007). A reactive tabu search algorithm with variable clustering for the unicost set covering problem. International Journal of Operational Research, 2(2), 156172. 
[16] Glover, F., \& McMillan, C. (1986). The general employee scheduling problem. An integration of MS and AI. Computers \& operations research, 13(5), 563-573. [5] Linn, R., \& Zhang, W. (1999). Hybrid flow shop scheduling: a survey. Computers \& industrial engineering, 37(1-2), 57-61.

[17] Glover, F., McMillan, C., \& Novick, B. (1985). Interactive decision software and computer graphics for architectural and space planning. Annals of operations research, 5(3), 557-573.

[18] Hansen, P., Jaumard, B., \& Da Silva, E. (1991). Average-linkage divisive hierarchical clustering. Cahiers du GERAD.

[19] Dorndorf, U., \& Pesch, E. (1994). Fast clustering algorithms. ORSA Journal on Computing, 6(2), 141-153.

[20] Glover, F., \& Taillard, E. (1993). A user's guide to tabu search. Annals of operations research, 41(1), 1-28.

[21] Beyer, D. A., \& Ogier, R. G. (1991, November). Tabu learning: a neural network search method for solving nonconvex optimization problems. In [Proceedings] 1991 IEEE International Joint Conference on Neural Networks (pp. 953-961). IEEE.

[22]Ganesh, S., \& Amutha, R. (2010). Real Time and Energy Efficient Transport Protocol for Wireless Sensor Networks.International Journal of Advanced Networking and Applications. arXiv preprint arXiv:1006.2691.

[23]Basavaraj, G. N., \& Jaidhar, C. D. (2019). Intersecting Sensor Range Cluster-based Routing Algorithm for Enhancing Energy in WSN. International Journal of Advanced Networking and Applications, 10(4), 39383943.

\section{Author's Biography}

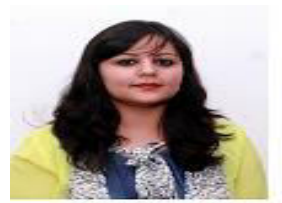

Varsha received her B-Tech from MIMIT, Malout, India and Master of Technology in Computer Science and Engineering from Guru Nanak Dev Engineering college ,Ludhianain the year 2011 and pursuing her Ph.D. from Punjab Technical University Jalandhar. She has published many papers in IEEE Conferences \& Scopus Indexed Journals and many more in the International/National Conferences. Her area of interest is Wireless Sensor Network, Data Communication and Computer Network.

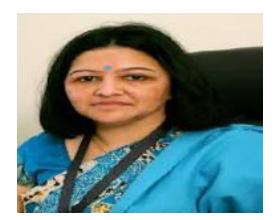

Manju Sharma received her B-Tech from UP Technical University, Lucknow, India and Master of Technology in Computer Science and Engineering from Punjab Technical University Jalandhar, in the year 2007 and she has doneher Ph.D. from Punjab Technical University Jalandhar. Currently, she is director at Khalsa College of Engineering \& Technology, Amritsar Punjab, India. She has published many papers in IEEE Conferences \&Scopus Indexed Journals and many more in the International/National Conferences. Her area of interest is Wireless Sensor Network, Data Communication and Computer Network. She is guiding many research scholars.

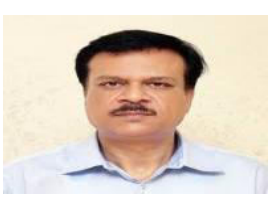

Manoj Kumar received his B.E (ECE) from Gulbarga University in the year 1990 \& received his M-Tech (ECE) from Punjab Technical University, Jalandhar in the year 2001 \&Ph.D. from Punjab Technical University Jalandhar in the year 2007. His area of specialization is Optical fibreCommunication, Wireless Communication. Currently $\mathrm{He}$ is a director at DAV Institute of Engineering \& Technology, Jalandhar. He has visited many Countries like U.S.A, U.K,Franceand Italy. He is life member of ISTE \& Punjab Academy of Sciences. 